\title{
Mesenchymal stem cells enhance the oncolytic effect of Newcastle disease virus in glioma cells and glioma stem cells via the secretion of TRAIL
}

\author{
Gila Kazimirsky ${ }^{1}$, Wei Jiang ${ }^{2}$, Shimon Slavin ${ }^{3}$, Amotz Ziv-Av ${ }^{1}$ and Chaya Brodie ${ }^{1,2^{*}}$ (B)
}

\begin{abstract}
Background: Newcastle disease virus (NDV) is an avian paramyxovirus, which selectively exerts oncolytic effects in cancer cells. Mesenchymal stem cells (MSCs) have been reported to affect tumor growth and deliver anti-tumor agents to experimental glioblastoma (GBM). Here, we explored the effects of NDV-infected MSCs derived from different sources, on glioma cells and glioma stem cells (GSCs) and the mechanisms involved in their effects.

Methods: The glioma cell lines (A172 and U87) and primary GSCs that were generated from GBM tumors were used in this study. MSCs derived from bone marrow, adipose tissue or umbilical cord were infected with NDV (MTH-68/H). The ability of these cells to deliver the virus to glioma cell lines and GSCs and the effects of NDVinfected MSCs on cell death and on the stemness and self-renewal of GSCs were examined. The mechanisms involved in the cytotoxic effects of the NDV-infected MSCs and their influence on the radiation sensitivity of GSCs were examined as well.

Results: NDV induced a dose-dependent cell death in glioma cells and a low level of apoptosis and inhibition of self-renewal in GSCs. MSCs derived from bone marrow, adipose and umbilical cord that were infected with NDV delivered the virus to co-cultured glioma cells and GSCs. Conditioned medium of NDV-infected MSCs induced higher level of apoptosis in the tumor cells compared with the apoptosis induced by their direct infection with similar virus titers. These results suggest that factor(s) secreted by the infected MSCs sensitized the glioma cells to the cytotoxic effects of NDV. We identified TRAIL as a mediator of the cytotoxic effects of the infected MSCs and demonstrated that TRAIL synergized with NDV in the induction of cell death in glioma cells and GSCs. Moreover, conditioned medium of infected MSCs enhanced the sensitivity of GSCs to $\gamma$-radiation.
\end{abstract}

Conclusions: NDV-infected umbilical cord-derived MSCs may provide a novel effective therapeutic approach for targeting GSCs and GBM and for sensitizing these tumors to $\gamma$-radiation.

Keywords: Newcastle disease virus (NDV), Glioblastoma (GBM), Glioma stem cells (GSCs), Mesenchymal stem cells (MSCs), TRAIL, y-radiation, Apoptosis, Self-renewal

\footnotetext{
*Correspondence: chaya@brodienet.com

'Mina \& Everard Goodman Faculty of Life-Sciences, Bar-llan University,

Ramat-Gan, Israel

${ }^{2}$ Hermelin Brain Tumor Center, Department of Neurosurgery, Henry Ford

Hospital, 2799 W Grand Blvd, Detroit, Ml 48202, USA

Full list of author information is available at the end of the article
} 


\section{Background}

Glioblastoma (GBM) remains one of the most malignant disorders in man with an average survival of 14 months despite optimal surgery, radiation therapy and temozolomide, which are considered the standard treatment of choice $[1,2]$. Due to the infiltrative/invasive nature of GBM and the complexity of the brain anatomy tumors cannot be completely removed in the large majority of cases [2]. GBMs contain a small population of glioma stem cells (GSCs) that exhibit treatment resistance which prevents a complete eradication of tumors cells and is associated with tumor recurrence [3, 4]. Therefore, new approaches for targeting resistant glioma cells and GSCs are urgently indicated to improve the prognosis of patients with GBM.

One of the novel approaches for the selective targeting of tumor cells is based on the use of oncolytic viruses $[5,6]$. These treatments combined specific tumor cell lysis by the viruses together with acting as in situ tumor vaccine [6]. Indeed, the potential use of oncolytic viruses for the treatment of cancer has been reported by several investigators with documentation of long-term survival of patients considered fully resistant to other available anti-cancer modalities [7]. Oncolytic viruses such as herpes simplex virus [8], vaccinia virus [9] and polio virus [10], have been reported as effective and selective therapies in GBM. In addition, there have been a number of reports indicating that Newcastle disease virus (NDV) also acts as an oncolytic virus in a number of tumors including GBM [11-16]. NDV is a well-known poultry virus with anti-neoplastic properties [17]. The preferential oncolytic activity of NDV toward malignant compared to normal cells is not fully understood, but has been attributed in part to reduced interferon secretion by malignant cells in contrast to normal cells [18]. Other mechanisms associated with the anti-tumor activity of NDV were also reported such as activation of the intrinsic death pathway, activation of the endoplasmic eIF2a kinase PERK and caspase 12, and the secretion of tumor necrosis factor alpha (TNF$\alpha)$ or TNF-related apoptosis-inducing ligand (TRAIL) from the infected tumor cells [7, 19-22]. Indeed, TRAIL has been considered as a promising anti-tumor agent with a strong clinical therapeutic potential [23, 24] and various studies demonstrated selective apoptotic effects of TRAIL on tumor cells including glioma cells and GSCs [25-29].

Administration of oncolytic viruses in clinical trials for GBM involves intratumoral or intravenous injections, which are associated with inefficient virus delivery [30]. One of the alternative approaches that have been explored for efficient virus delivery is the use of stem cells, such as mesenchymal stem cells (MSCs), as delivery vehicles. MSCs exhibit homing abilities to sites of injury, inflammation and tumors [31-34]. Specifically, MSCs have been shown to migrate to sites of experimental GBMs and to deliver cytotoxic compounds that exert anti-tumor effects [31, 32]. MSCs can be obtained from autologous bone marrow (BM) and adipose (AD) tissues [35, 36] or from allogeneic placenta and umbilical cord, which then can be used as "off-theshelf" cells [37, 38]. These cells can be easily expanded in vitro and used safely for various therapeutic indications $[39,40]$. Recent studies suggest that despite sharing similar cell surface markers, MSCs that are derived from different sources exhibit differences in their transcriptome, cytokine profile and biological effects [35]. Therefore, MSCs from various sources can have different therapeutic impacts in specific clinical indications.

In view of the broad and selective anti-cancer properties of NDV [41, 42] and the fact that MSCs migrate actively to tumor sites and cells including cancer stem cells, we investigated the potential therapeutic effect of NDV-infected MSCs on glioma cells and GSCs.

\section{Methods \\ GSC cultures}

All human materials were used in accordance with the policies of the institutional review board at Henry Ford Hospital, Detroit, MI, USA. The generation of the GSCs and their characterization were recently described [43-45]. Briefly, GBM specimens were dissociated in $0.05 \%$ Trypsin/EDTA for $4 \mathrm{~h}$ at room temperature followed by mechanical dissociation. Cells were maintained in neurosphere medium supplemented with $20 \mathrm{ng} / \mathrm{ml}$ epidermal growth factor (EGF) and $20 \mathrm{ng} / \mathrm{ml}$ basic fibroblast growth factor (FGF-beta) and were examined for the expression of the stemness markers, CD44, Bmi-1, CD133, Musashi-1, Sox 2 and nestin and self-renewal. All the GSCs employed in this study were examined for tumorigenic potential in nude mice or rats as recently reported [43-45].

\section{Mesenchymal stem cell cultures}

Bone marrow (BM)-derived MSCs, adipose tissue (AD)derived MSCs, and umbilical cord (UC) tissue-derived MSCs were obtained from ScienCell Research Laboratories (Carlsbad, CA, USA) and were characterized and maintained as previously described [46]. The cells expressed CD73, CD90 and CD105 and were negative for CD14, CD34, CD80 and CD45. The different cell types were also examined for their ability to differentiate to osteoblasts, chondrocytes and adipocytes. The purity of all the MSC preparations was over $95 \%$.

\section{Co-culture experiments}

For the co-culture experiments, MSCs and glioma cells or GSCs were plated in transwell plates with a $0.4-\mu \mathrm{m}$ filter. In some experiments, conditioned medium of infected 
MSCs was isolated and administered directly to the GSCs or glioma cells.

\section{NDV infection}

Oncolytic NDV (MTH-68) prepared at the Beit Dagan Institute, Israel was used in all experiments. Cells were infected with different titers of NDV for $2 \mathrm{~h}$, after which the cells were washed three times and incubated with fresh medium.

\section{Real-time PCR analysis}

Total RNA was isolated from cultured cells using QIAzol reagent (Qiagen, Valencia, CA, USA) according to the manufacturer's protocol. A total of $0.5 \mu \mathrm{g}$ of RNA was employed to synthesize cDNA by Thermoscript (Invitrogen, Carlsbad, CA, USA) with oligodT primers. Primers, $25 \mu \mathrm{L}$ of $2 \times$ SYBR Green Master Mix (Invitrogen), and 30-100 ng cDNA samples were resuspended in a total volume of $50 \mu \mathrm{L}$ PCR amplification solution. Reactions were run on an ABI Prism 7000 Sequence Detection System (Applied Biosystems, Foster City, CA, USA). Cycle threshold $(\mathrm{Ct})$ values were obtained from the ABI 7000 software. The following primers were used: NDV: 5'-TCA CAGACTCAACTCTTGGG-3' and 5'-CAGTATGAGGT GTCAAGTTCTTC-3' as reported [47]. S12 expression was determined for each RNA sample as a control.

\section{Self-renewal assay}

The formation of secondary neurospheres by GSCs was measured in cells that were plated in 24-well plates at a density of 10 cells/well through limiting dilution. The number of neurospheres/well was determined 2 weeks later for ten different wells. Spheres that contained more than 20 cells were scored as described [43-45].

\section{Cell death assays}

Three methods were employed to analyze cell death: (1) measurements of lactate dehydrogenase (LDH) levels in culture supernatants, (2) caspase3/7 activity, and (3) expression of total and cleaved PARP by Western blot analysis. Caspase-3/7 activity in the GSCs was measured using a caspase-3/7 assay kit (Promega, Madison, WI, USA) according to the manufacturer's instructions.

\section{Western blot analysis}

Western blot analysis was performed as described. Equal loading was verified using an anti- $\beta$-actin or tubulin antibodies as described $[45,46]$.

\section{TRAIL secretion and neutralization}

The concentrations of TRAIL ligand in cell supernatants were measured using a commercial ELISA kit from Diaclone Research (Besancon, France), according to the manufacturer's instructions. A TRAIL neutralizing antibody (Abcam, Cambridge, MA, USA; $5 \mu \mathrm{g} / \mathrm{ml}$ )), was added in some of the experiments $24 \mathrm{~h}$ prior to the addition of the conditioned medium. A corresponding isotype-matched antibody was used as a control.

\section{Statistical analysis}

The results are presented as the mean values \pm standard error of the mean (SE). Data were analyzed using analysis of variance or a Student's $t$ test with correction for data sets with unequal variances.

\section{Results}

NDV exerts selective oncolytic effects on glioma cells and GSCs

We first examined the oncolytic effects of NDV on glioma cell lines and GSCs. Cells were infected with increasing titers of NDV and cell death was examined after 24 and 48 h. As presented in Fig. 1a, NDV induced cell death in both U87 and A172 glioma cell lines already in 1 multiplicity of infection (MOI) and plateau levels were obtained at $5 \mathrm{MOI}$ for both cell lines. In contrast, infection of human astrocytes with 10 MOI of NDV induced only a small degree of cell death (Fig. 1a). Morphological analysis of the infected cells demonstrated similar results - increased cell death in the infected U87 cells with no differences in the cell morphology of human astrocytes (Fig. 1a).

Although NDV has been reported to exert potent oncolytic effects on cancer cells, its effects on cancer stem cells or GSCs has not been described. We therefore examined the oncolytic effect of NDV on GSCs obtained from fresh glioma specimens that were previously described and reported by us [43, 44, 46, 48]. In these studies, we employed the two GSCs HF2355 and HF2359 and examined the effects of NDV infection on the self-renewal and cell death of these cells. We found that NDV induced cytotoxic effects on both GSCs albeit to a different degree (Fig. 1c) as determined by LDH assay and by PARP cleavage for the HF2359 cells (Fig. 1d). For both GSCs, NDV exerted a lower cytotoxic effect compared to the glioma cell lines. Similar results were obtained for an additional two GSCs (data not shown). In contrast, no significant cytotoxic effect was observed in human neural stem cells (NSCs) even at 10 MOI and after $72 \mathrm{~h}$ (Fig. 1c).

The cytotoxic effect of NDV was also observed on the stemness characteristics of the GSCs including smaller neurosphere size (Fig. 1e) and inhibition of self-renewal of these cells (Fig. 1f). Using secondary neurosphere formation assay, we found that after 10 days NDV at MOI of 1 significantly decreased the neurosphere size (Fig. 1e) and the self-renewal of the GSCs (Fig. 1f). 

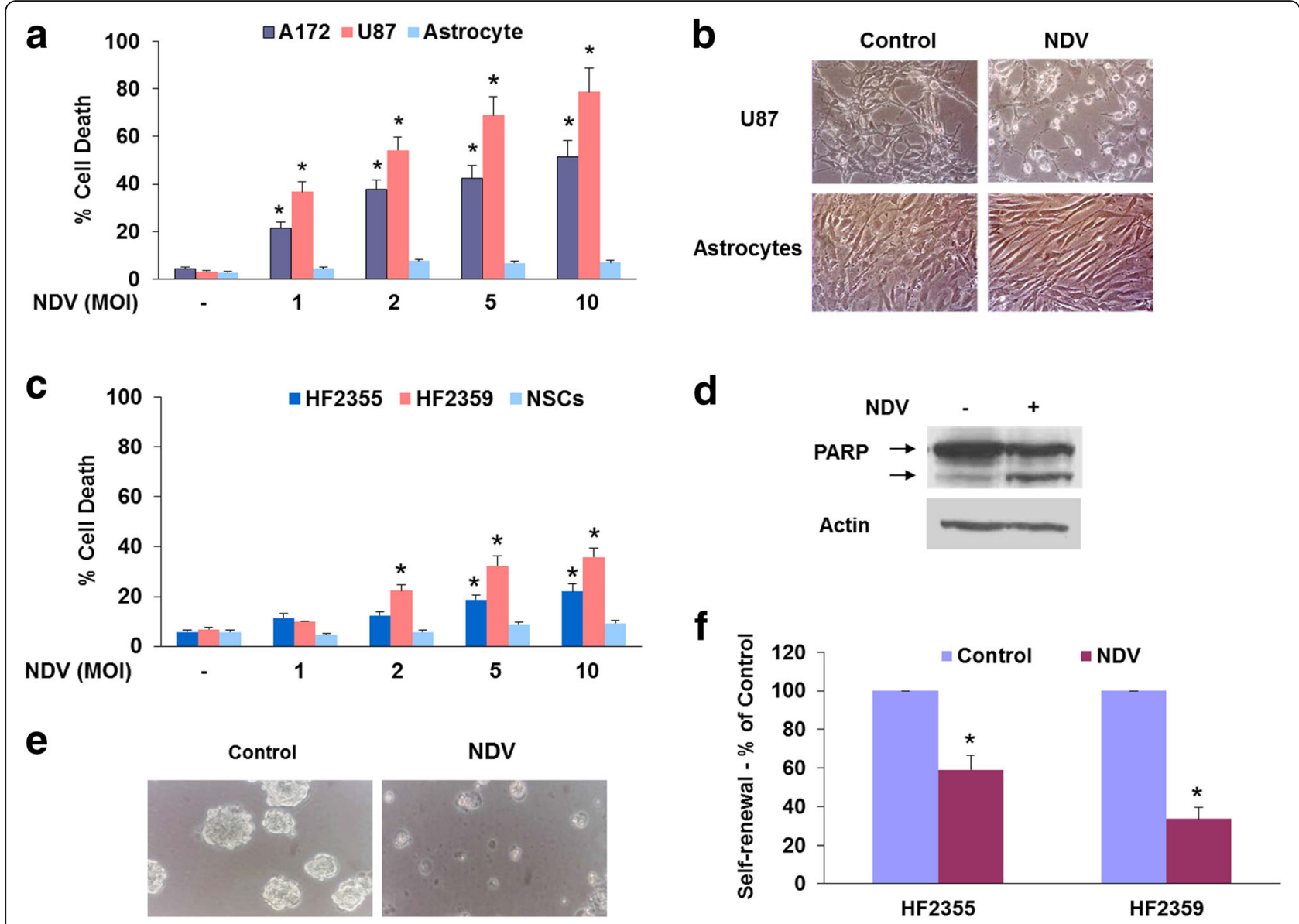

Fig. 1 NDV induces a selective cell death in glioma cells and glioma stem cells. The glioma cell lines, U87 and A172 or human astrocytes were infected with different titers of NDV and cell death was determined using LDH release into the culture supernatants after $48 \mathrm{~h}$ (a). The morphology of U87 cells and human astrocytes was analyzed following NDV infection (2 MOI) using phase contrast microscopy (b). Cell death was also analyzed in two GSC cultures and human NSCs using LDH assay (c) and in the HF2355 cells using Western blot analysis of cleaved PARP expression (d). Infection with NDV induced disaggregation of the GSC spheroids (e). The self-renewal of the infected GSCs was determined after 14 days of infection (1 MOI) (f). The results are presented as means \pm SE and represent three different experiments $(\mathbf{a}, \mathbf{c}) .{ }^{*} p<0.001$ (control vs. infected cells). One representative of three similar experiments is presented (b, d-f). MOI multiplicity of infection, NDV Newcastle disease virus, NSC neural stem cell

\section{Conditioned medium of NDV-infected MSCs enhances the} virus cytotoxic effect

MSCs have been reported to deliver oncolytic viruses to various tumors including glioma [16]. To examine the ability of MSCs to deliver NDV to glioma cells we first analyzed the infection of the different MSCs by NDV. For these experiments, we employed MSCs derived from $\mathrm{BM}, \mathrm{AD}$ and umbilical cord (UC) tissues. We found that infection of the MSCs with NDV induced some cell death after 4 days (around 25-40\%, depending on the MSC source, Fig. 2a) and a more pronounced effect after 5 days (data not shown).

We then examined the ability of MSCs to deliver NDV to glioma cells or GSCs using co-cultures plated in transwell plates with a $0.4-\mu \mathrm{m}$ filter that does not allow cell transfer or by using MSC-conditioned medium.
We first demonstrated that MSCs were able to deliver NDV to co-cultured glioma cells, as indicated by the detection of NDV in the glioma cells using qRT-PCR (Fig. 2b). We did not find a significant difference in the ability of the different MSCs to increase NDV expression in the glioma cells. Similar results were obtained with co-cultured GSCs (data not shown).

As presented in Fig. 2c, co-culturing of glioma cells with MSCs infected with NDV induced a larger degree of cell death in the glioma cells compared to cells directly infected with the same virus titer. Thus, infection of A172 cells with 2 MOI NDV induced about $20 \%$ cell death, whereas co-culturing of A172 cells with NDV-infected BM-MSCs induced over $40 \%$ cell death. Similar results were obtained also with AD-MSCs. 


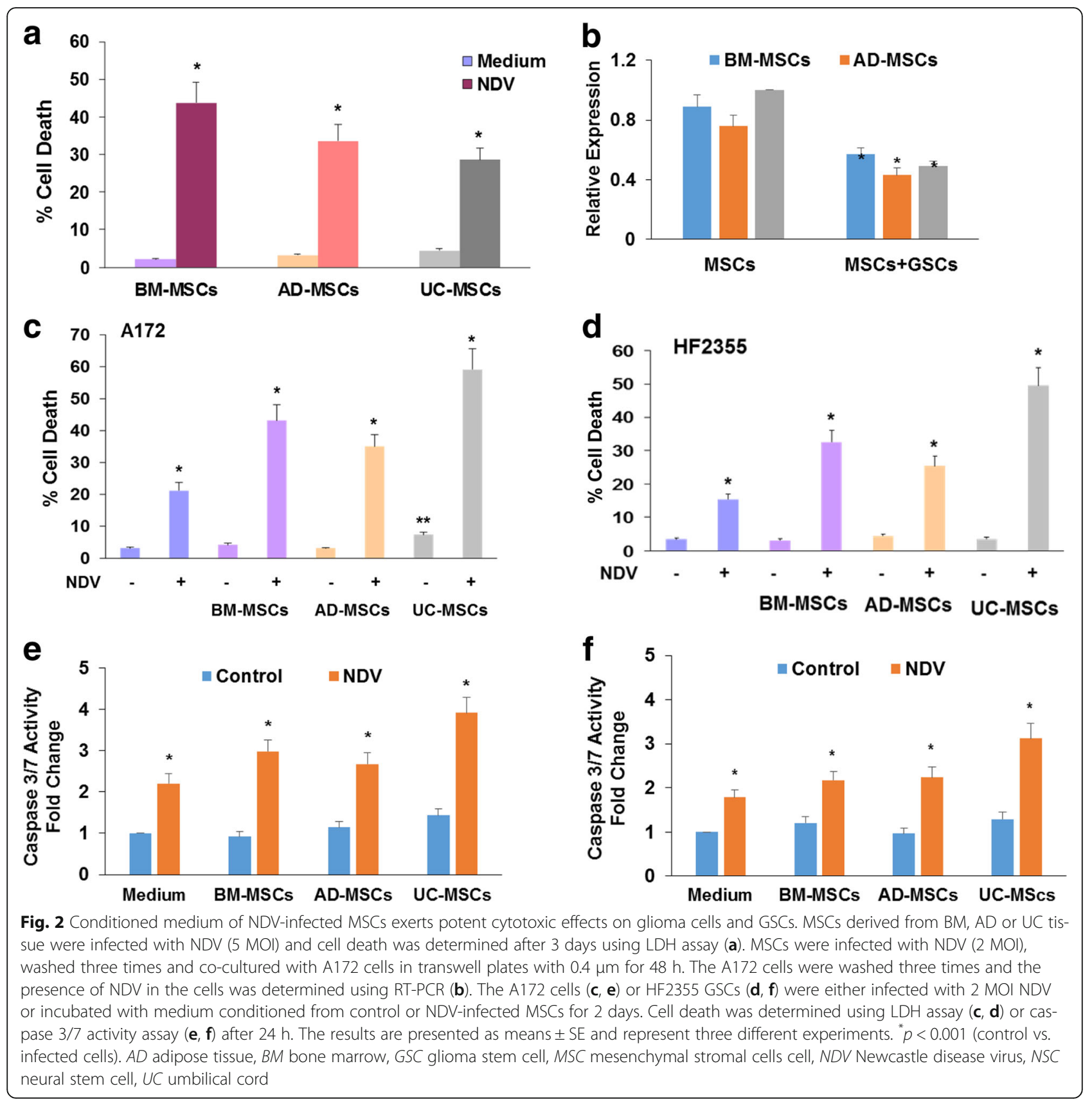

Interestingly UC-MSCs infected with NDV induced the largest cytotoxic effect of glioma cells as compared with the other types of MSCs. Similar effects were observed with conditioned medium of the infected MSCs (data not shown). The increased cytotoxic effect of conditioned medium derived from NDV-infected MSCs was also observed in GSCs as compared to their lowered response to direct infection with NDV (Fig. 2d). Similar effects were obtained with measurements of caspase $3 / 7$ activity in both A172 cells (Fig. 2e) and the HF2355 GSCs (Fig. 2f).
The increased cytotoxic effect of NDV-infected MSCs is mediated by TRAIL

We next analyzed the factors that mediate the enhanced cytotoxic effects of NDV-infected MSCs on glioma cells and GSCs. Using ELISA of infected MSC-conditioned medium we found that control UC cells secreted low levels of TRAIL and that all infected MSCs secreted increased TRAIL levels, with UC-MSCs demonstrating the highest levels (Fig. 3a).

To further examine if the increased TRAIL secretion mediated the enhanced cytotoxic effect of the infected 


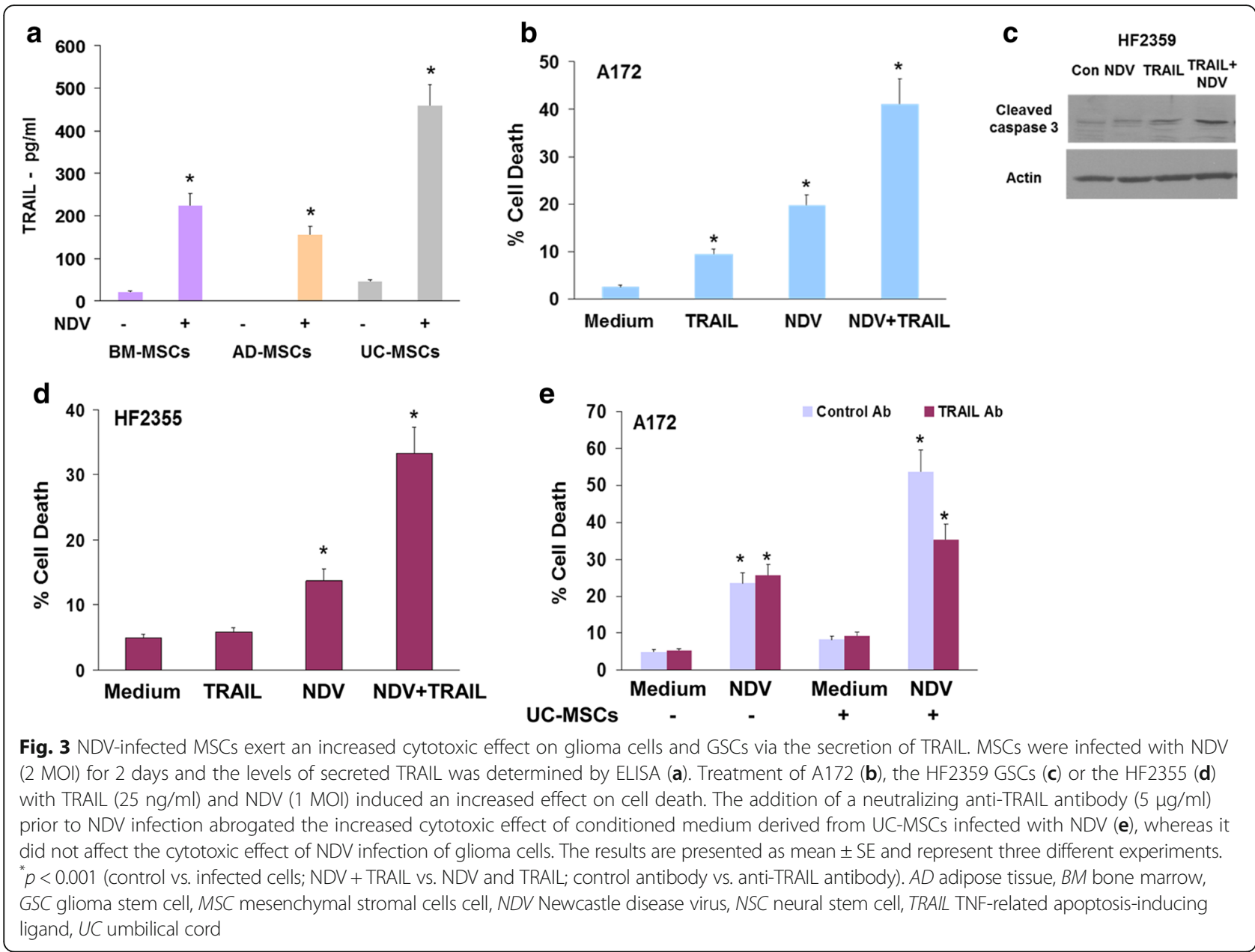

MSCs we first analyzed the combined effect of TRAIL and NDV on cell apoptosis of glioma cells and GSCs. As demonstrated in Fig. 3b, NDV (1 MOI) sensitized glioma cells to the apoptotic effects of low concentrations of TRAIL $(25 \mathrm{ng} / \mathrm{ml})$, that by itself induced only a marginal degree of cell death (Fig. 3b). Similarly, NDV also sensitized the GSCs, HF2359 (Fig. 3c) and HF2355 (Fig. 3d) to the apoptotic effects of TRAIL. A neutralizing antiTRAIL antibody partially inhibited the increased cytotoxic effect of conditioned medium of NDV-infected UC-MSCs on A172 cells (Fig. 3e), suggesting that TRAIL secreted by the infected cells mediated this increased effect. Similar results were obtained with the HF2355 and HF2359 GSCs (data not shown).

In contrast, human astrocytes and the NSCs did not exhibit significant cell death in response to either TRAIL, conditioned medium of NDV-infected UC-MSCs or the combination of both treatments (data not shown).

Altogether, these results implicate TRAIL as an important factor secreted by infected MSCs that can augment the cytotoxic effect of NDV on glioma cells and GSCs.

\section{Conditioned medium from NDV-infected MSCs sensitize glioma stem cells to $\gamma$-radiation}

$\gamma$-radiation is the first-line treatment for GBM patients; however, GSCs have been reported to exhibit increased resistance to this treatment [49]. Combined treatment of GSCs with $\gamma$-radiation and TRAIL induced increased cytotoxic effects $[27,50]$. We therefore examined the effect of conditioned medium of NDV-infected MSCs on the response of GSCs to $\gamma$-radiation. As presented in Fig. 4a, the HF2355 GSCs exhibited a small decrease in self-renewal in response to $\gamma$-radiation treatment (3 Gy) and this response was further increased in NDV-infected cells (1 MOI). Similar results were also obtained with the HF2414 and HF2359 GSCs (data not shown). The effects of $\gamma$-radiation and NDV on cell death in these cells were very modest (Fig. 4b). In contrast, we found that a combined treatment of conditioned medium from UC-MSCs infected with NDV and $\gamma$-radiation exerted a pronounced effect on cell death as compared to each treatment alone and as was measured by LDH assay (Fig. 4d) and caspase3/7 activity (Fig. 4c). These results suggest that conditioned medium derived from NDV- 


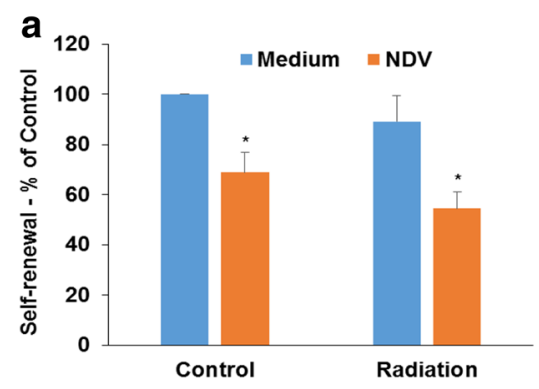

C

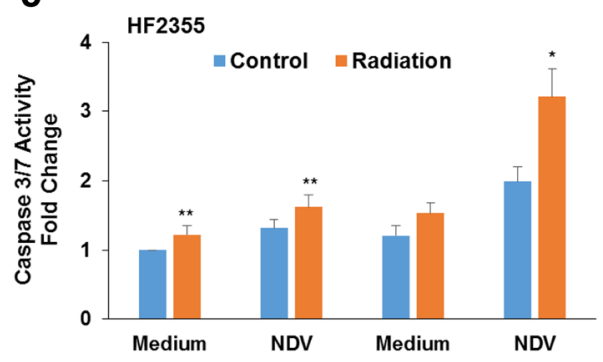

b
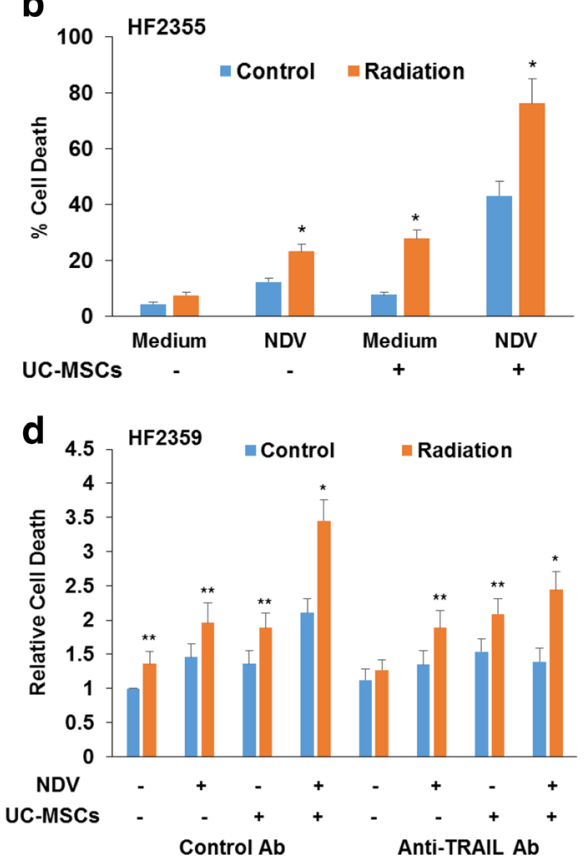

Fig. 4 Combined treatment of GSCS with conditioned medium derived from NDV-infected UC-MSCs and radiation exerts a synergistic cytotoxic effect on GSCs. The HF2355 GSCs were infected with NDV and irradiated (3 Gy) (a). The self-renewal of the cells was determined after 10 days of treatment. HF2355 GSCs were irradiated in the presence or absence of conditioned medium derived from NDV-infected UC-MSCS (b, c). Cell death was determined after 3 days using LDH (b) or caspase 3/7 (c) assays. The role of TRAIL in the increased sensitization to radiation was examined in the HF2359 GSCs. The addition of a neutralizing anti-TRAIL antibody (5 $\mathrm{\mu g} / \mathrm{ml})$ prior to NDV infection and $\gamma$-irradiation abrogated the increased cytotoxic effect of the infected UC-MSCs conditioned medium and that of the combined effects of $\gamma$-radiation and the conditioned medium. Cell death was measured using LDH assay and data are presented as relative cell death (d). The results are presented as the means \pm SE and represent three different experiments. ${ }^{*} p<0.001 ;{ }^{* *} p<0.01$. ( ${ }^{*}$ Control vs. infected cells; radiation + CM of NDV-infected UC-MSCs vs. radiation or NDV alone; control vs. anti-TRAIL antibody; ${ }^{* *}$ GSCs treated with UC-MSC CM and NDV infected GSCs vs. untreated cells). MSC mesenchymal stromal cells cell, NDV Newcastle disease virus, TRAIL TNF-related apoptosis-inducing ligand, UC umbilical cord

infected MSCs can sensitize GSCs to the cytotoxic effects of $\gamma$-radiation.

We also examined if TRAIL secreted by the NDVinfected UC-MSCs mediated the increased cytotoxic effect of UC-MSC conditioned medium and $\gamma$-radiation. Using the neutralizing anti-TRAIL antibody that was employed in the experiments presented in Fig. 3e, we demonstrated that the secretion of TRAIL by the NDVinfected UC-MSCs played at least a partial role in enhancing the response of the GSCs to $\gamma$-radiation (Fig. 4d).

\section{Discussion}

In this study, we examined the effects of NDV-infected MSCs from different sources on glioma cells and GSCs and the mechanisms involved in their effects.

NDV has been reported to induce selective apoptosis of various cancer cell lines including glioma [11, 14, 16, 51]; however, its effects on human astrocytes have not been examined. We demonstrated that infection of U87, U251 and A172 cells with NDV induced cell apoptosis already at $1 \mathrm{MOI}$ and that normal human astrocytes were resistant to the cytotoxic effect of NDV even at 10 MOI, further suggesting that NDV effects are tumor cell selective.

GBMs contain a population of GSCs that contribute to therapy resistance and tumor recurrence despite successful surgical removal of visible tumor [52]. Thus, identifying treatments that can selectively target these cells is of utmost importance in the treatment of GBM. Our results show for the first time that NDV can also target GSCs and that although these cells are less sensitive than differentiated tumors cells to the cytotoxic effect of NDV, they exhibit a decreased self-renewal ability when infected with low virus titers. Importantly, also for these cells, the effects of NDV appear to be tumor selective and human NSCs are resistant to NDV infection as indicated in Fig. 1c.

MSCs have been reported to migrate to tumor sites, such as glioma, and to deliver various anti-cancer treatments including oncolytic viruses [31, 32, 53-55]. Our results indicate that MSCs can be also used to deliver oncolytic NDV to glioma cells and GSCs. We found that NDV infected the different MSCs and that these cells 
underwent apoptosis after 4 days of infection and in response to higher virus titers as compared to glioma cells. These results suggest that NDV-infected MSCs can deliver the virus effectively and can be used safely for the eradication of NDV-sensitive GSCs with no residual presence of MSCs that could theoretically inhibit the local immune response elicited by the infected cells $[5,30]$.

Interestingly, treatment of glioma cell lines and GSCs with conditioned medium of NDV-infected MSCs induced a larger cytotoxic effect as compared to glioma cells infected with similar NDV titers. The most pronounced effect was obtained with UC-MSC infected cells compared with BM and AD-derived MSCs. We found that the infected UC-MSCs secreted high levels of TRAIL and that treatment of glioma cells with conditioned medium of NDV-infected UC-MSCs in the presence of anti-TRAIL neutralizing antibody abrogated the enhanced cytotoxic effect of the conditioned medium. Moreover, infection of glioma cells and GSCs with NDV sensitized these cells to the apoptotic effect of TRAIL, further supporting our conclusion that this factor mediated at least some of the enhanced cytotoxic effects of the NDV-infected MSCs on glioma cells.

TRAIL has been reported to induce a selective cell apoptosis in tumor cells and has been considered a promising anti-tumor agent $[23,29]$. Indeed, multiple studies demonstrated the apoptotic effect of TRAIL on a variety of tumor cells including glioma cells [25-28, 56]. Despite the selective effects of TRAIL on tumor cells there are some cells (in particular cancer stem cells) that exhibit resistance to the apoptotic effect of this ligand [57]. Thus, the current results that demonstrate an increased cytotoxic effect of TRAIL and NDV in both glioma cells and GSCs may provide a mechanism to bypass the relative resistance of GSCs to both TRAIL and NDV. Indeed, a recent study demonstrated enhanced anti-tumor effects of NDV engineered to express TRAIL and IL-2 [58, 59]. The delivery of TRAIL by MSCs engineered to overexpress this protein was recently reported to exert cytotoxic effects in glioma xenografts $[60,61]$. Our findings demonstrate a novel approach to increase the secretion of endogenous TRAIL by NDV infection of UC-MSCs, which may further enhance the anti-tumor effects of these cells as was recently reported [62, 63].

Our results of sensitizing GSCs to $\gamma$-radiation by the conditioned medium of NDV-infected MSCs demonstrated a mechanism to overcome the resistance of GSCs to radiation, which is one of the first-line treatments for GBM. Indeed, a combined treatment of TRAIL and $\gamma$ radiation has been reported to exert a synergistic effect in vitro and in vivo [50]. Importantly, recent studies demonstrated that radiation increased the homing of
MSCs to glioma xenografts [50], therefore suggesting that not only NDV-infected MSCs are expected to home to the tumor site more efficiently, but they can also enhance the response of resistant tumor cells to $\gamma$ radiation.

Considering the preferential migration of MSCs to tumor sites, it seems reasonable to hypothesize that using MSCs as a delivery system is likely to provide an effective method for the selective targeting of NDV to glioma cells and GSCs. Thus, encapsulating NDV in MSCs can overcome the current limitations in virus delivery and lack of extravasion into the tumor and can shield the viruses from sequestration in the liver and neutralization. Since treatment of GBM patients with NDV seems to be effective in a small fraction of cases [64], it seems reasonable to assume that a more efficient transfer of NDV into the tumor cells may result in a more effective anti-cancer impact for a larger number of patients. Thus, a targeted delivery of NDV by MSCs may represent a substantially more effective approach for transfer of NDV into the malignant cells, rather than relying on random delivery of the circulating viruses.

Preliminary clinical studies suggest that treatment with NDV is safe [16]. Similarly, recent preclinical and clinical studies using both autologous and allogeneic MSCs from various sources indicate that treatment of patients with various neurological and inflammatory disorders is safe and has some therapeutic impact [65-69]. Based on these studies and our results showing the lack of toxicity of NDV or MSCs loaded with NDV on normal astrocytes and NSCs, we expect that treatment with MSCs loaded with NDV is also likely to be harmless against normal neural cells in vivo. Thus, infection of MSCs with NDC may represent a novel approach for the treatment of minimal residual disease and the eradication of GSCs following or along with conventional modalities.

\section{Conclusions}

The infection of UC-MSCs with oncolytic viruses such as NDV can provide a new therapeutic strategy by combining the homing ability of MSCs towards tumor sites and the increased secretion of factors with anti-tumor activity such as TRAIL from the infected cells. Thus, using NDV-infected MSCs is expected to result in targeted delivery of the virus to tumor cells and enhanced anti-tumor effects in both glioma cells and GSCs. In addition, the presence of the infected cells may enhance the local immune response at the tumors sites as was reported for cells infected with other viruses [70]. NDVinfected umbilical cord-derived MSCs may therefore provide a novel effective therapeutic approach for targeting GSCs and GBM and for sensitizing these tumors to $\gamma$-radiation. 


\section{Abbreviations}

AD: Adipose tissue; BM: Bone marrow; EGF: Epidermal growth factor; FGF: Fibroblast growth factor; GBM: Glioblastoma; GSC: Glioma stem cell; LDH: Lactate dehydrogenase; MOI: Multiplicity of infection; MSC: Mesenchymal stromal cells cell; NDV: Newcastle disease virus; NSC: Neural stem cell; TNF-a: Tumor necrosis factor alpha; TRAIL: TNF-related apoptosis-inducing ligand; UC: Umbilical cord

\section{Acknowledgements}

We wish to thank Dr. Shimon Perg from the Kimron Veterinary Institute at Beit Dagan, the diagnostic and research arm of the Ministry of Agriculture Veterinary Services and Animal Health Unit, Israel for providing oncolytic NDV.

\section{Funding}

This work is supported by the William and Karen Davidson Fund, the Hermelin Brain Tumor Center, by the Lori and Alan Zekelman Fund, by the Association for Cancer Therapy and Transplantation Medicine, Tel-Aviv, Israel and by International Clinical Research Fellowship (ICRF) (CB).

\section{Availability of data and materials}

Not applicable

\section{Authors' contributions}

GK and AZA performed the experiment, analyzed the data and contributed to the drafting of the manuscript. WJ analyzed the data and prepared the manuscript. SS designed some of the experiments and contributed to the manuscript drafting. CB designed the experiments, analyzed the data and wrote the manuscript. All authors read and approved the manuscript.

\section{Competing interests}

The authors declare that they have no competing interests.

\section{Consent for publication}

All human materials were used in accordance with the policies of the institutional review board at Henry Ford Hospital, Detroit, MI, USA.

\section{Ethical approval and consent to participate}

Not applicable.

\section{Author details}

1 Mina \& Everard Goodman Faculty of Life-Sciences, Bar-llan University, Ramat-Gan, Israel. ${ }^{2}$ Hermelin Brain Tumor Center, Department of Neurosurgery, Henry Ford Hospital, 2799 W Grand Blvd, Detroit, MI 48202, USA. ${ }^{3}$ Hadassah Medical Center, Hebrew University, Jerusalem, Israel.

Received: 1 July 2016 Revised: 12 September 2016 Accepted: 16 September 2016 Published online: 10 October 2016

\section{References}

1. Stupp R, Hegi ME, Mason WP, van den Bent MJ, Taphoorn MJ, et al. Effects of radiotherapy with concomitant and adjuvant temozolomide versus radiotherapy alone on survival in glioblastoma in a randomised phase III study: 5-year analysis of the EORTC-NCIC trial. Lancet Oncol. 2009;10(5):459-66.

2. Burton EC, Prados MD. Malignant gliomas. Curr Treat Options Oncol. 2000; 1(5):459-68.

3. Singh SK, Hawkins C, Clarke ID, Squire JA, Bayani J, et al. Identification of human brain tumour initiating cells. Nature. 2004:432(7015):396-401.

4. Vescovi AL, Galli R, Reynolds BA. Brain tumour stem cells. Nat Rev Cancer. 2006;6(6):425-36.

5. Parker JN, Bauer DF, Cody JJ, Markert JM. Oncolytic viral therapy of malignant glioma. Neurotherapeutics. 2009;6(3):558-69.

6. Chiocca EA, Rabkin SD. Oncolytic viruses and their application to cancer immunotherapy. Cancer Immunol Res. 2014;2(4):295-300.

7. Elankumaran S, Chavan V, Qiao D, Shobana R, Moorkanat G, et al. Type I interferon-sensitive recombinant Newcastle disease virus for oncolytic virotherapy. J Virol. 2010;84(8):3835-44.

8. Kaur B, Chiocca EA, Cripe TP. Oncolytic HSV-1 virotherapy: clinical experience and opportunities for progress. Curr Pharm Biotechnol. 2012; 13(9):1842-51.
9. Lun X, Chan J, Zhou H, Sun B, Kelly JJ, et al. Efficacy and safety/toxicity study of recombinant vaccinia virus JX-594 in two immunocompetent animal models of glioma. Mol Ther. 2010;18(11):1927-36.

10. Brown MC, Gromeier M. Cytotoxic and immunogenic mechanisms of recombinant oncolytic poliovirus. Curr Opin Virol. 2015;13:81-5.

11. Cassel WA, Garrett RE. Newcastle disease virus as an antineoplastic agent Cancer. 1965;18:863-8.

12. Csatary LK. Viruses in the treatment of cancer. Lancet. 1971;2(7728):825.

13. Csatary LK, Moss RW, Beuth J, Torocsik B, Szeberenyi J, et al. Beneficial treatment of patients with advanced cancer using a Newcastle disease virus vaccine (MTH-68/H). Anticancer Res. 1999;19(1B):635-8.

14. Sinkovics JG, Horvath JC. Newcastle disease virus (NDV): brief history of its oncolytic strains. J Clin Virol. 2000;16(1):1-15.

15. Webb HE, Smith CE. Viruses in the treatment of cancer. Lancet. 1970; 1(7658):1206-8.

16. Zamarin D, Palese P. Oncolytic Newcastle disease virus for cancer therapy: old challenges and new directions. Future Microbiol. 2012;7(3):347-67.

17. Nemunaitis J. Live viruses in cancer treatment. Oncology (Williston Park). 2002;16(11):1483-92. discussion 1495-1487.

18. Krishnamurthy S, Takimoto T, Scroggs RA, Portner A. Differentially regulated interferon response determines the outcome of Newcastle disease virus infection in normal and tumor cell lines. J Virol. 2006;80(11):5145-55.

19. Elankumaran S, Rockemann D, Samal SK. Newcastle disease virus exerts oncolysis by both intrinsic and extrinsic caspase-dependent pathways of cell death. J Virol. 2006:80(15):7522-34.

20. Lorence RM, Rood PA, Kelley KW. Newcastle disease virus as an antineoplastic agent: induction of tumor necrosis factor-alpha and augmentation of its cytotoxicity. J Natl Cancer Inst. 1988;80(16):1305-12.

21. Washburn B, Weigand MA, Grosse-Wilde A, Janke M, Stahl H, et al. TNFrelated apoptosis-inducing ligand mediates tumoricidal activity of human monocytes stimulated by Newcastle disease virus. J Immunol. 2003;170(4): 1814-21.

22. Zorn U, Dallmann I, Grosse J, Kirchner H, Poliwoda H, et al. Induction of cytokines and cytotoxicity against tumor cells by Newcastle disease virus. Cancer Biother. 1994;9(3):225-35.

23. Johnstone RW, Frew AJ, Smyth MJ. The TRAIL apoptotic pathway in cancer onset, progression and therapy. Nat Rev Cancer. 2008;8(10):782-98.

24. Lim B, Allen JE, Prabhu W, Talekar MK, Finnberg NK, et al. Targeting TRAIL in the treatment of cancer: new developments. Expert Opin Ther Targets. 2015:19(9):1171-85.

25. Okhrimenko H, Lu W, Xiang C, Hamburger N, Kazimirsky G, et al. Protein kinase $\mathrm{C}$-epsilon regulates the apoptosis and survival of glioma cells. Cancer Res. 2005;65(16):7301-9.

26. Okhrimenko H, Lu W, Xiang C, Ju D, Blumberg PM, et al. Roles of tyrosine phosphorylation and cleavage of protein kinase Cdelta in its protective effect against tumor necrosis factor-related apoptosis inducing ligandinduced apoptosis. J Biol Chem. 2005:280(25):23643-52.

27. Perlstein B, Finniss SA, Miller C, Okhrimenko H, Kazimirsky G, et al. TRAIL conjugated to nanoparticles exhibits increased anti-tumor activities in glioma cells and glioma stem cells in vitro and in vivo. Neuro Oncol. 2013; 15(1):29-40.

28. Kahana S, Finniss S, Cazacu S, Xiang C, Lee HK, et al. Proteasome inhibitors sensitize glioma cells and glioma stem cells to TRAlL-induced apoptosis by PKCepsilon-dependent downregulation of AKT and XIAP expressions. Cell Signal. 2011:23(8):1348-57.

29. Kim Y, Seol DW. TRAlL, a mighty apoptosis inducer. Mol Cells. 2003;15(3): 283-93.

30. Wollmann G, Ozduman K, van den Pol AN. Oncolytic virus therapy for glioblastoma multiforme: concepts and candidates. Cancer J. 2012;18(1):69-81.

31. Altaner C, Altanerova V, Cihova M, Ondicova K, Rychly B, et al. Complete regression of glioblastoma by mesenchymal stem cells mediated prodrug gene therapy simulating clinical therapeutic scenario. Int J Cancer. 2014; 134(6):1458-65.

32. Vogel $\mathrm{S}$, Peters $\mathrm{C}$, Etminan $\mathrm{N}$, Borger $\mathrm{V}$, Schimanski $\mathrm{A}$, et al. Migration of mesenchymal stem cells towards glioblastoma cells depends on hepatocyte-growth factor and is enhanced by aminolaevulinic acidmediated photodynamic treatment. Biochem Biophys Res Commun. 2013:431(3):428-32.

33. Zhang R, Liu Y, Yan K, Chen L, Chen XR, et al. Anti-inflammatory and immunomodulatory mechanisms of mesenchymal stem cell transplantation in experimental traumatic brain injury. J Neuroinflammation. 2013;10:106. 
34. lyer SS, Rojas M. Anti-inflammatory effects of mesenchymal stem cells: novel concept for future therapies. Expert Opin Biol Ther. 2008;8(5):569-81.

35. Jin HJ, Bae YK, Kim M, Kwon SJ, Jeon HB, et al. Comparative analysis of human mesenchymal stem cells from bone marrow, adipose tissue, and umbilical cord blood as sources of cell therapy. Int J Mol Sci. 2013;14(9): 17986-8001.

36. Lazarus HM, Haynesworth SE, Gerson SL, Rosenthal NS, Caplan Al. Ex vivo expansion and subsequent infusion of human bone marrow-derived stromal progenitor cells (mesenchymal progenitor cells): implications for therapeutic use. Bone Marrow Transplant. 1995;16(4):557-64.

37. Hass R, Kasper C, Bohm S, Jacobs R. Different populations and sources of human mesenchymal stem cells (MSC): a comparison of adult and neonatal tissue-derived MSC. Cell Commun Signal. 2011;9:12.

38. Vellasamy S, Sandrasaigaran P, Vidyadaran S, George E, Ramasamy R. Isolation and characterisation of mesenchymal stem cells derived from human placenta tissue. World J Stem Cells. 2012;4(6):53-61.

39. Caplan Al. Why are MSCs therapeutic? New data: new insight. J Pathol. 2009;217(2):318-24.

40. Slavin S, Kurkalli BG, Karussis D. The potential use of adult stem cells for the treatment of multiple sclerosis and other neurodegenerative disorders. Clin Neurol Neurosurg. 2008;110(9):943-6.

41. Pap M, Bator J, Szeberenyi J. Sensitivity of human malignant melanoma cell lines to Newcastle disease virus. Anticancer Res. 2015;35(10):5401-6.

42. Shobana R, Samal SK, Elankumaran S. Prostate-specific antigen-retargeted recombinant newcastle disease virus for prostate cancer virotherapy. J Virol. 2013;87(7):3792-800

43. Bier A, Giladi N, Kronfeld N, Lee HK, Cazacu S, et al. MicroRNA-137 is downregulated in glioblastoma and inhibits the stemness of glioma stem cells by targeting RTVP-1. Oncotarget. 2013;4(5):665-76.

44. deCarvalho AC, Nelson K, Lemke N, Lehman NL, Arbab AS, et al. Gliosarcoma stem cells undergo glial and mesenchymal differentiation in vivo. Stem Cells. 2010;28(2):181-90.

45. Lomonaco SL, Finniss S, Xiang C, Decarvalho A, Umansky F, et al. The induction of autophagy by gamma-radiation contributes to the radioresistance of glioma stem cells. Int J Cancer. 2009;125(3):717-22.

46. Lee HK, Finniss S, Cazacu S, Bucris E, Ziv-Av A, et al. Mesenchymal stem cells deliver synthetic microRNA mimics to glioma cells and glioma stem cells and inhibit their cell migration and self-renewal. Oncotarget. 2013;4(2):346-61.

47. Liu N, Long Y, Liu B, Yang D, Li C, et al. ISG12a mediates cell response to Newcastle disease viral infection. Virology. 2014;462-463:283-94.

48. Giladi ND, Ziv-Av A, Lee HK, Finniss S, Cazacu S, et al. RTVP-1 promotes mesenchymal transformation of glioma via a STAT-3/IL-6-dependent positive feedback loop. Oncotarget. 2015;6(26):22680-97.

49. Cheng $\mathrm{L}$, Bao S, Rich JN. Potential therapeutic implications of cancer stem cells in glioblastoma. Biochem Pharmacol. 2010;80(5):654-65.

50. Kim SM, Oh JH, Park SA, Ryu CH, Lim JY, et al. Irradiation enhances the tumor tropism and therapeutic potential of tumor necrosis factor-related apoptosis-inducing ligand-secreting human umbilical cord blood-derived mesenchymal stem cells in glioma therapy. Stem Cells. 2010:28(12):2217-28

51. Reichard KW, Lorence RM, Cascino CJ, Peeples ME, Walter RJ, et al. Newcastle disease virus selectively kills human tumor cells. J Surg Res. 1992;52(5):448-53.

52. Lathia JD, Mack SC, Mulkearns-Hubert EE, Valentim CL, Rich JN. Cancer stem cells in glioblastoma. Genes Dev. 2015;29(12):1203-17.

53. Dembinski JL, Spaeth EL, Fueyo J, Gomez-Manzano C, Studeny M, et al. Reduction of nontarget infection and systemic toxicity by targeted delivery of conditionally replicating viruses transported in mesenchymal stem cells. Cancer Gene Ther. 2010;17(4):289-97.

54. Garcia-Castro J, Alemany R, Cascallo M, Martinez-Quintanilla J, Arriero Mde $M$, et al. Treatment of metastatic neuroblastoma with systemic oncolytic virotherapy delivered by autologous mesenchymal stem cells: an exploratory study. Cancer Gene Ther. 2010;17(7):476-83.

55. Nakashima H, Kaur B, Chiocca EA. Directing systemic oncolytic viral delivery to tumors via carrier cells. Cytokine Growth Factor Rev. 2010;21(2-3):119-26.

56. Rahman M, Pumphrey JG, Lipkowitz S. The TRAlL to targeted therapy of breast cancer. Adv Cancer Res. 2009;103:43-73.

57. Capper D, Gaiser T, Hartmann C, Habel A, Mueller W, et al. Stem-cell-like glioma cells are resistant to TRAIL/Apo2L and exhibit down-regulation of caspase-8 by promoter methylation. Acta Neuropathol. 2009;117(4):445-56.
58. Bai F, Niu Z, Tian H, Li S, Lv Z, et al. Genetically engineered Newcastle disease virus expressing interleukin 2 is a potential drug candidate for cancer immunotherapy. Immunol Lett. 2014;159(1-2):36-46.

59. Bai $F L, ~ Y u ~ Y H$, Tian $H$, Ren GP, Wang $H$, et al. Genetically engineered Newcastle disease virus expressing interleukin-2 and TNF-related apoptosisinducing ligand for cancer therapy. Cancer Biol Ther. 2014;15(9):1226-38.

60. Kim SM, Lim JY, Park SI, Jeong CH, Oh JH, et al. Gene therapy using TRAILsecreting human umbilical cord blood-derived mesenchymal stem cells against intracranial glioma. Cancer Res. 2008;68(23):9614-23.

61. Menon LG, Kelly K, Yang HW, Kim SK, Black PM, et al. Human bone marrowderived mesenchymal stromal cells expressing S-TRAIL as a cellular delivery vehicle for human glioma therapy. Stem Cells. 2009;27(9):2320-30.

62. Akimoto K, Kimura K, Nagano M, Takano S, To'a Salazar G, et al. Umbilical cord blood-derived mesenchymal stem cells inhibit, but adipose tissuederived mesenchymal stem cells promote, glioblastoma multiforme proliferation. Stem Cells Dev. 2013;22(9):1370-86.

63. Velpula KK, Dasari VR, Tsung AJ, Dinh DH, Rao JS. Cord blood stem cells revert glioma stem cell EMT by down regulating transcriptional activation of Sox2 and Twist1. Oncotarget. 2011;2(12):1028-42.

64. Freeman Al, Zakay-Rones Z, Gomori JM, Linetsky E, Rasooly L, et al. Phase I/II trial of intravenous NDV-HUJ oncolytic virus in recurrent glioblastoma multiforme. Mol Ther. 2006:13(1):221-8.

65. Park SJ, Moon SH, Lee HJ, Lim JJ, Kim JM, et al. A comparison of human cord blood- and embryonic stem cell-derived endothelial progenitor cells in the treatment of chronic wounds. Biomaterials. 2013;34(4):995-1003.

66. Gao LR, Pei XT, Ding QA, Chen Y, Zhang NK, et al. A critical challenge: dosage-related efficacy and acute complication intracoronary injection of autologous bone marrow mesenchymal stem cells in acute myocardial infarction. Int J Cardiol. 2013;168(4):3191-9.

67. Martinez-Morales PL, Revilla A, Ocana I, Gonzalez C, Sainz P, et al. Progress in stem cell therapy for major human neurological disorders. Stem Cell Rev. 2013:9(5):685-99.

68. Zhang $\mathrm{R}$, Chen $\mathrm{H}$, Zheng Z, Liu Q, Xu L. Umbilical cord-derived mesenchymal stem cell therapy for neurological disorders via inhibition of mitogen-activated protein kinase pathway-mediated apoptosis. Mol Med Rep. 2015;11(3):1807-12.

69. Karussis D, Karageorgiou C, Vaknin-Dembinsky A, Gowda-Kurkalli B, Gomori JM, et al. Safety and immunological effects of mesenchymal stem cell transplantation in patients with multiple sclerosis and amyotrophic lateral sclerosis. Arch Neurol. 2010;67(10):1187-94.

70. Tong AW, Senzer N, Cerullo V, Templeton NS, Hemminki A, et al. Oncolytic viruses for induction of anti-tumor immunity. Curr Pharm Biotechnol. 2012; 13(9):1750-60.

\section{Submit your next manuscript to BioMed Central and we will help you at every step:}

- We accept pre-submission inquiries

- Our selector tool helps you to find the most relevant journal

- We provide round the clock customer support

- Convenient online submission

- Thorough peer review

- Inclusion in PubMed and all major indexing services

- Maximum visibility for your research

Submit your manuscript at www.biomedcentral.com/submit
) Biomed Central 